\title{
Test and evaluation protocols for GPR-based mine-detection systems; a proposal
}

\author{
Jan B. Rhebergen ${ }^{a}$ and James M. Ralston ${ }^{b}$ \\ ${ }^{a}$ TNO Defense, Security \& Safety (TNO-DS\&S) The Hague, Netherlands; \\ ${ }^{b}$ Intsitute for Defense Analyses (IDA), Alexandria, VA. U.S.
}

\begin{abstract}
With the emergence of commercially available multisensor mine detection systems, the need for standardised test and evaluation procedures becomes more pressing. For metal detectors this already has been established and is laid down in the CEN workshop document CWA14747:2003 [1]. The ITEP multisensor working group has taken the first step towards a similar document, by means of a so called "best practice" document, which would ultimately lead to a proper standard. In this paper we address various issues important for multisensor mine detection testing and evaluation and in this way hope to contribute to a draft version of the "best practice" document encouraging other parties to do the same and hence speed up the process of standardisation.
\end{abstract}

Keywords: landmines, GPR, MD, multisensor, testing, evaluation, protocols, procedures

\section{INTRODUCTION}

Over the past year or so the demining community has witnessed the emergence of a few commercially available hand held multisensor mine detection system targeted as a replacement or addition to the more conventional metal detector based mine detection system. The well known problem facing deminers using metal detectors is the huge number of false alarms. These multisensor systems aim to reduce the amount of false alarms considerably and hence speed up the demining process. Because of this projected speed gain, a higher unit price of these technologically advanced systems may be acceptable.

Even though some companies already had multisensor vehicle based systems commercially available, hand-held systems systems were often either lab models still in development, or prototypes. The advent of relatively cheap RF and DSP components as by products of the mobile communications market has sparked the latest round of development and has propelled it forward into a commercialisation phase. Although costs are still much higher than traditional detection means, the gap narrows and, as indicated earlier, increased performance may offset higher cost.

Given the current developments, the "International Test and Evaluation Program" for Humanitarian Demining (ITEP) decided to form the "Multisensor Working Group" (MSWG) in February of 2004. Representatives of the ITEP member countries contribute in many ways to the efforts of this working group by cooperating and participating in, in-country tests or by drafting test procedure proposals etc. In fact the current article is an attempt to contribute in the same spirit. The ITEP-MSWG has set itself the following goals:

- Review information on existing test-facilities.

- Highlight those suitable in principle for multisensor testing purposes.

- Contribute to list of existing test reports on multisensor detectors [2].

- Facilitate infrastructure for sharing measurement data, information on specialist test equipment, etc. between participants.

- Define first steps to a test methodology including test protocols.

- Define the needs of multisensor test environment.

Further author contact information: E-mail: jan.rhebergen@tno.nl, Telephone: +31 703740772 
- Conduct initial tests with existing multisensor systems (prototypes or commercial).

- Track emerging multisensor mine detection technologies.

- Review facilities and protocols on the basis of emerging technologies/developments.

- Draft initial version of protocol/procedure leading up to guidelines (best practice).

Depending the perspective one is taking, the procedure for testing and evaluation may emphasise different aspects, because different perspectives dictate different needs or demands. One could make the following distinction:

- User perspective:

- Buyer: government or non-governmental organisations.

- Seller: developers, manufacturers, researchers.

- End user: humanitarian or military de-miner.

- Technological perspective:

- Test facilities.

- Test methodology and protocols.

These perspectives intersect and overlap to a certain degree. In this paper we will attempt to contribute to a "best practice" proposal from the perspective of the end user and also somewhat from the perspective of the researcher (akin to the technological perspective). The main areas of attention will be limited to:

- Systems (GPR, MD, IR, plus others).

- Environment (soil, EM, weather).

- Test objects, scenarios and setup.

- Methodology

- Evaluation (and presentation) of results.

\section{SYSTEMS}

Some companies already have had multisensor vehicle based systems available for a number of years e.g. Geo-Centers EF-GPR [3] (also includes MD and IR), and more recently NIITEK Wichmann GPR. Others are just entering the arena e.g. several Japanese systems under the coordination of the "Japan Science and Technology" (JST) agency [4].

Some of the older systems, originally developed for AT-mine detection, already have had some opportunity to be tested under simulated field conditions and have now been updated to also find AP-mines. The more recent systems are just beginning to be tested. Apart from tests under simulated field conditions we now also see in-country testing in locations like, Thailand, Cambodia, Afghanistan, Angola, Bosnia, etc. This is especially true for the emerging new commercial of the shelf hand-held multisensor mine detection systems, from Cyterra/HSTAMIDS (US) and ERA Minehound (UK) which are in the process of being tested and commercialised. In the remainder of this paper we will mostly focus on hand-held multisensor systems which consist of a metal detector in combination with the ground penetrating radar.

So far these test have not been standardised although manufacturers try to follow similar procedures. Results are difficult to compare because so much depends on the actual testing and environmental conditions. Apart from that systems are often in different stages of development making a fair comparison unrealistic. Even if systems and conditions are comparable manufacturers may be reluctant to pit their products against each other. Nevertheless, in order to draft sensible testing procedures and protocols manufacturers input is also needed. 


\subsection{Specifications}

Depending on your perspective you may want or need to verify the equipment performance, specification and functionality as claimed by the manufacturer of the equipment you are intending to test. Given a specific, properly operating, minedetection system, the end user (deminer) will be more concerned with functional issues such as battery life, size, weight, standoff reach, display brightness or audibility, etc than with RF power output or waveform bandwidth.

Verifying basic specifications, as stated by manufacturer, can serve several purposes. Failure to meet specifications, can point to a malfunction in the equipment before testing. Secondly the equipment under test may need to conform to EMC/EMI criteria that allow for other equipment, also needed for testing (or mine-detection), to function properly. Typical systems specifications to verify are for instance:

- Power output

- Dynamic range

- Signal to noise ratio

- Bandwidth

- Sensor 'footprint'

- Battery life

Ideally these specifications should all be checked with in-air setups.

\subsection{Functionality and capability}

The most important and most difficult to test is the detection performance of the system. Probably foremost on the mind of the deminer is the question of whether the system he is using actually performs its function well. If not, he will not dare enter the minefield. A suitable built-in go/no-go test to verify basic functionality before using the equipment is an absolute must. The operator needs to have confidence in his equipment. One could think of a small on site in-air or even in-ground test.

To summarise, basic detection capability should be verified right before use, while detailed performance can be tested in a more controlled surrounding well before actual use. Functionality can be verified in a simple fashion by using a typical (real) target on-site and determine the detection in an in-air setup. The same can also be executed with an in-soil scenario using the same ground and typical depths that occur at the site that is going to be surveyed.

Detection performance should be determined as a function of:

- target depth and size

- soil type

- soil moisture content e.g.

- dry ca. $5 \%$

- damp ca. $20 \%$

- wet ca. $40 \%$ or more

- target material and shape 


\section{ENVIRONMENT}

The biggest influence on the performance of a multisensor system is the environment in which it is tested and/or subsequently operated. Because many unknown parameters characterise the environment, to fully characterise the environment would be impractical. Therefore one should concentrate on those that exercise the most influence on the performance.

Obviously the ground is the main component of the environment. However, especially if one also uses additional sensors like infrared cameras, one might want to employ a mini weather station to monitor above ground meteorological conditions. At the very least one should probably record subjectively observed conditions by the testers or deminers on site and obtain records of the weather conditions from a local weather service.

Apart from the above mentioned, the equipment also has to function in the local electromagnetic environment. Typically multisensor mine-detectors are designed not to have soil contact (for obvious reasons), although it would be better for their performance if they did. The fact that they are suspended above the air/ground interface makes them more vulnerable to outside interference. Presence of power lines, cellphones, or other in-band transmitters affect the performance negatively. Usually manufacturers have taken measures to ensure that the effects are minimised. Nevertheless operators and testers need to be aware of these vulnerabilities and take them into account. For tests sites interference sources are usually known and for active demining sites they should be noted as well.If possible operations should be conducted only when interfering equipment is switched off.

Multisensor mine-detection systems that contain a GPR emit electromagnetic radiation in a frequency band that may interfere with many other devices. Cell phones and GPS receivers or other communications devices may be vulnerable. Current systems based on commercially available GPR are subject to strict emissions standards. Governments and regulatory authorities may need to either lift or impose less stringent norms for GPR systems that are used as part of mine detection systems.

In general for each sensor in a multisensor system one should carefully consider what influence the environment has on its performance. We have shown some possible considerations for GPR, MD and IR, the same can of course be done for acoustic or other sensors as well.

\subsection{Soil environment}

A lot is known about the effects of the soil environment on metal detectors and ground penetrating radars. For metal detectors the main parameter that determines the performance is the soil magnetic (complex) susceptibility while for the ground penetrating radar the complex permittivity generally is more important. The latter depends strongly on the soil water content, with higher water content leading to higher losses. Hence determining soil water content (apart from soil composition and type) is very important. On the other hand, if the dielectric contrast between mine and soil under dry conditions is very low, a modest amount of moisture increases the contrast, [5] improving detectability by GPR. Higher permittivity also decreases the wavelength in the soil enhancing the target resolution. An added advantage of soil with a higher dielectric contrast could be that the direct (surface reflected) wave may be better separated in time from the reflections of objects in the ground. To summarise, the soil electromagnetic propagation characteristics like soil conductivity $\sigma$ from $30 \mathrm{~Hz}$ to $1 \mathrm{MHz}$ and soil attenuation and propagation factors $\alpha, \beta$ from $100 \mathrm{MHz}$ to $3 \mathrm{GHz}$, should be determined. These values can be found by sending a hermetically sealed and carefully collected soil sample to a specialised laboratory. Some labs, i.e. at Delft University, are able to measure these values with a prescribed soil water content over a very broad frequency range.

\subsubsection{Soil composition \& type}

Characterisation of the soil as appropriate for the tests should shed light on parameters like:

- sand fraction,

- clay fraction,

- bulk density,

- organic matter,

- bulk soil texture 
to name but a few. Most of these parameters can be obtained by sending samples off to a soil analysis lab. The soil is dried, sieved, and treated in other ways to determine a histogram of grain sizes, percentage dry matter and organic matter, etc. When the soil composition is determined one can subsequently classify it according to a commonly used soil texture map as shown in figure 3.1.1 [6] One of the main reasons for determining the soil composition is to be able to reconstruct later

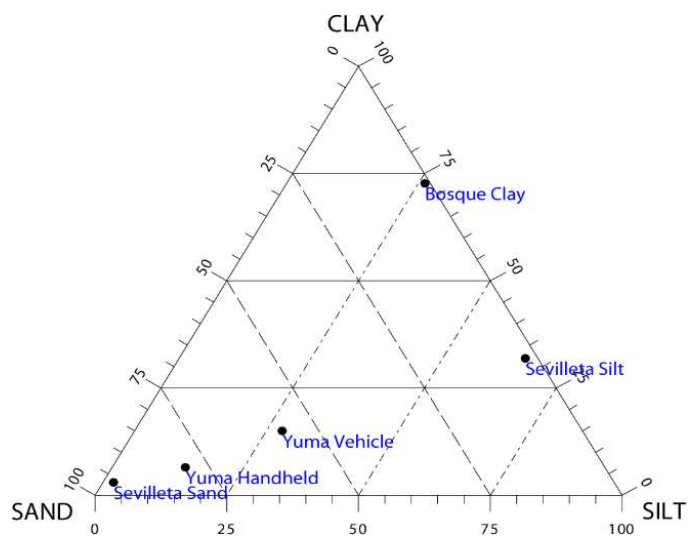

Figure 1. USDA soil texture classification with some example entries

under what conditions a particular test or demining activity was executed and then hopefully compare results or even dare to predict future detector performance given these conditions. The advantage will only become apparent after a substantial number of test (or demining activities) have been executed at different sites. Because of this, end users and manufacturers may not be very enthusiastic about gathering this kind of information, viewing it as a extra burden.

\subsubsection{Soil moisture}

The soil moisture or water content plays a complex role in detection performance. As indicated earlier it can act in counterintuitive ways. A little bit of moisture may under certain condition actually be advantageous depending on conditions. For instance if the soil moisture is not evenly distributed in the ground, e.g. puddles, dry spots or moisture 'fingers', the general clutter level is increased and probability of false detections increases. A moderate homogeneous increase in soil moisture may actually increase the target contrast and be beneficial to detection probability. These effects are hard to predict and model although some general conclusions may be drawn e.g. sudden or extreme variations should be avoided. At a test site one can try to limit the variation in soil water content, or even control it to a certain extend. The TNO demining test site [7] has preset water tables which can be set to almost any desired level within a certain range. Normally the levels are set to what would be typical for a certain soil type and desired scenario. Being an outside open air facility, the amount of control is of course limited. Screens to shield the test lanes from the most extreme conditions are available. The added advantage of these screens is that the lack of light slows down the growth of weeds and such considerably. Influence of vegetation is a whole chapter in itself and is not addressed further in this paper.

Of course the soil water content is intimately linked with the actual and historic weather conditions at test site or demining areas. Having a log or report of weather conditions at the time of operation is valuable information. During a demining operation it is advantageous to avoid (if possible) periods of extreme weather conditions.

Measurements of the soil water content can be taken by means of time domain reflectometry (TDR) although other means are also possible e.g. frequency domain and capacitance measurements. The following issues should be addressed.

- Soil water content $\Theta$ as a function of depth i.e. a profile. Ideally several measurements over a depth of up to 30 to $50 \mathrm{~cm}$ centimetre should be taken (depending on the scenario).

- Soil water content $\Theta$ averaged over an area. This can be achieved by averaging a few spot measurements spread out over a larger area.

- Proper operation of calibrated equipment is important to get reliable readings (i.e. first prod with a stylus slightly smaller than the TDR probe) 
Hermetically sealed samples from several depths can be sent to a lab for analysis of water content by means of heating. This way TDR measurements are 'pegged' and instruments can be calibrated.

\subsubsection{Soil surface}

Most sensors are sensitive to variations in surface conditions. This is particularly true for GPR. Apart from the physical difficulty of negotiating a rough terrain the electromagnetic waves are scattered in an irregular way increasing clutter. The level of enhanced clutter is directly related to the operating frequency of the GPR and the dimensions of the surface irregularities. The surface roughness is generally random. The condition of the surface can be classified according to the following terms:

- rough/fine/smooth (mean correlation length)

- steps, (size)

- holes, (depth)

- undulations

Although a laser generated surface roughness map may not be necessary, photographs (with scales/dimensions) that document the typical surface conditions should be recorded.

\subsection{Site setup and layout}

Depending on whether hand held or vehicle mounted systems are going to be tested different needs will define the site setup and layout. Typically a test site for a vehicle based multisensor system may need to be much bigger covering an area of up to several hectares. If systems are not intimately linked with the carrying vehicle platform they may be removed and mounted on moving measurement bridge able to carry the same weight. The latter is the case at the TNO test facility [7].

The advantage of being able to mount multisensor systems on movable measurement bridges are:

- Stability

- Accuracy

- Repeatability

In the case of hand held systems the operator has a big influence on the overall performance of the system. Sufficient training to operate sensor competently is essential. Defining a proper measurement procedure and conduct with respect to the operator is a whole subject in itself (see human factors not covered here). If one really wants to objectively evaluate a multisensor system, one needs to exclude unknown or difficult to quantify parameters keeping the test as simple as possible with a few variables as needed. For hand-held systems it would be desirable to operate them using a mechanical/robotic scanning mechanism so that human performance factors such as operator fatigue and attention will not influence the test.

A good test site should address the following issues or have the following characteristics:

Contain a reference area: A small area set aside to verify proper system operation and calibrate sensors. This area should be partly empty (though big enough) and partly filled with a few well separated reference targets whose sensor responses are well known,

Test specific sensor parameters: Maximum detection depth, lateral resolution, battery life, object identification (if possible), data integrity, operational speed, warm-up time, drift, performance deviations.

Increase in difficulty: To be able to find the sensor performance 'envelope' the conditions should be gradually increased in difficulty. This can be in terms of more difficult environment (soil type, surface), more challenging target layout/scenarios. 


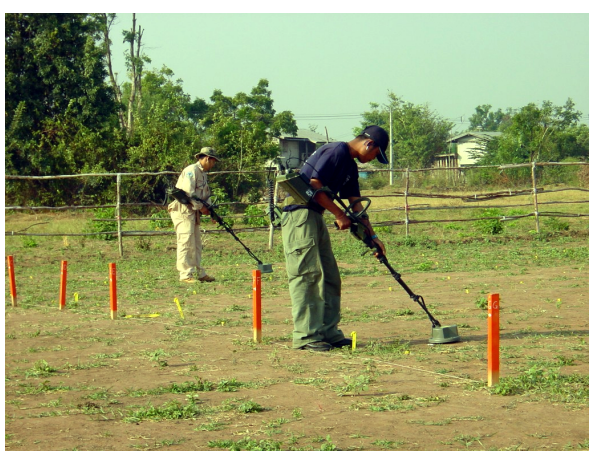

(a) In-country test site, Thailand

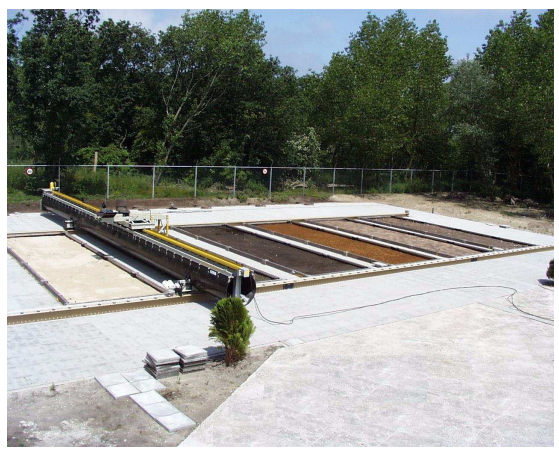

(b) Controlled test site, TNO

Figure 2. Test site layout examples

Mimic suitable conditions: These conditions should be realistic for sensors that are close to being fielded in realistic conditions. More controlled and somewhat less realistic conditions are valuable because they usually eliminate unknown or unwanted environmental variables.

Figure 2 shows a few examples of different test sites. The in-country test site has a very realistic environment but there is no control over soil type, soil water content etc. The TNO test site [7] tries to mimic reality to a certain degree. The different soils were taken from a natural environment and are disturbed as little as possible. The ground water table is controlled to what it would be in the natural setting. Permanently buried sensors continuously monitor temperature and soil moisture content.

\subsection{Test objects}

When a GPR detects an object this object can be either a land-mine, a non-land-mine object or a soil anomaly. It should be clearly defined what detection actually means. A test environment will contain objects for detections. These object should be representative of the targets that are normally found in the mined area (i.e. mines and false alarms). Apart from that certain reference targets should also be included. Any mine detection system offered for test will likely include specific data processing or fusion features intended to discriminate against clutter. One should ensure that the test scenario includes a full range of clutter objects to allow the value of such processing to be determined. Not only the type of target but also the configuration of targets in the test environment should be specified i.e. several objects grouped together with varying separation and burial depth.

\subsubsection{True targets}

In addition to the influence of soil type and moisture, we have seen evidence that GPR performance varies according to mine composition (low- or high-metal content), size (AP or AT), burial depth, and elapsed time since burial. Rather than trying to include all possible combinations of mine situations in statistically significant numbers, we should identify a range of cases, from simple to stressing.

Mine targets can be grouped into rough categories:

- Model, replica and training mines (superficially similar)

- Simulant and surrogate mines (similar in physical and EM characteristics, may contain fuse and booster simulants)

- Real inactive mines (contain explosives but lack detonators)

In recent discussion with manufacturers it has become apparent that there is a need for targets that are even closer to mines than the present simulants and surrogates. However these targets would probably be so similar to real mines that severe restrictions would be imposed upon their use. 


\subsubsection{False alarms}

False alarms can arise from specific man-made inclusions in soil (metal fragments, cans, other objects) as well as from naturally occurring inhomogeneities. While the former can be controlled in a test site, it is difficult to keep specific records on natural sources of false alarms. The condition of the test site should be carefully surveyed for any apparent:

- non-mine but mine-like objects

- strange objects, i.e. false alarms due to non-mine or mine-like objects,

- non-objects, i.e. false alarms that constitute soil bound anomalies like,

- soil macro structures

- water/moisture concentrations

- soil-type and density variation

\subsubsection{Reference targets}

This category includes specific target objects that are not intentionally mine-like. Their primary value lies in obtaining fundamental radar characteristics (response strength, spatial response footprint, etc) rather than aggregated detection performance. Some candidate reference targets include:

- ITOP object including casing (possibly modified)

- metal sphere of various sizes

- dielectric spheres

- flat plates or discs

Although there is quite a bit of agreement on what reference targets to include, one of the things that is still lacking is a good proposal for a reference background (apart form air).

\section{METHODOLOGY}

\subsection{General issues}

We need to fit the test procedure to the subject system's level of technological readiness and indicate this in describing the end result. A graduated course of tests with increasing difficulty could comprise:

- lab environment

- field conditions

- mine affected areas

Testing in a controlled lab environment would be the first step in characterising a single sensor, such as GPR or MD. More complex simulated field conditions would be important for evaluating multi sensor systems due to their dependence on differently varying environmental conditions. Initially these tests would not be conducted as 'blind' tests.Important radar characteristics to be evaluated in these initial controlled but not blind tests would include:

- depth/time resolution and response side lobes

- lateral spatial resolution (ability to distinguish nearby objects)

- antenna footprint (spatial area of adequate system response)

In addition to the radar's instrumental characteristics, detection performance can be described in terms of: 
- Probability of detection vs false alarm rate (ROC curve)

- Correlation of detection confidence levels with known object signatures.

- Ultimately blind field test (general methodology) would be required to assess these performance factors for both single or multisensor systems.

\section{2. multisensor issues}

Probably the most critical question to resolve in the testing of a fused metal detector/GPR mine detecting system is the performance increment provided by the addition of GPR. The issues to be addressed include:

- It is important to verify the measurable increase in the detection and/or decrease in FAR due to extra sensor.

- This should be tested with separate/combined sensors.

- We must track the dependence of performance on target difficulty (to what extent are deep/small mines more accessible with the combined sensor suite?)

- Define (limited set) environmental test scenarios of increasing difficulty. Some factors would include:

- Soil type and homogeneity.

- Presence/absence of typical inclusions

- Density of metal fragments (critical in systems that include a metal detector)

- Surface roughness

- Surface vegetation

\section{EVALUATION OF TEST RESULTS}

Evaluation of tests results should be executed as objective as possible. There are several ways to achieve this. One way is to have an outside party take care of this task. Another way is to automate the procedure so that any chance of 'contaminating' the results is minimised.

\section{Important sensor performance characteristics}

- Detection probability $\left(P_{d}\right)$ and FAR (i.e. ROC curve)

- lateral spatial resolution

- depth/time resolution (if applicable)

- Detection confidence levels (e.g. for fusion)

The ROC curve is a very widely used and accepted means of evaluating sensor performance. However it may obfuscate some important sensor characteristics that also play a part in the overall sensor performance [8]. Sometimes it may not be easy (or even impossible) to assess the ROC curve of a given sensor due to the fact that the sensor does not return an explicit alarm, but detection is based on operator interpretation of underground images. In this case one could contemplate applying a method described in [9] which also discusses a way to limit the number of experiments needed to get statistically significant answers. 


\section{Performance and scoring}

As part of the scoring procedure all the data has to be analysed before a ROC curve can be constructed. A familiar way to depict this process that also give a clear understanding of the issues can be seen in figure 3 Statistics derived from this are an important means to communicate performance next to the familiar ROC curve.

- Scoring similar to that used for MD or like that used for counter-mine experiments in the US, where scoring is based on software to minimise subjective factors.

- Differentiate between sensor in primary detection role or as confirmation sensor. As a primary detector, the coverage rate is most critical, while a secondary detector must have a low probability of false alarm at a high probability of detection, to avoid rejecting legitimate target declarations by the first sensor.

- Include results on blind and non-blind test (emphasis on former).

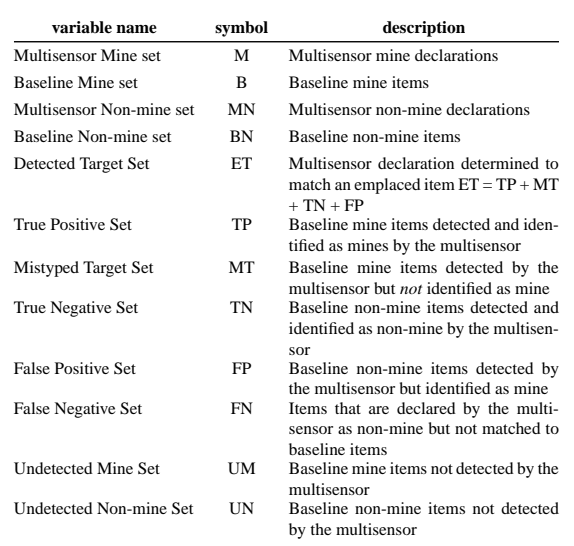

(a) terminology

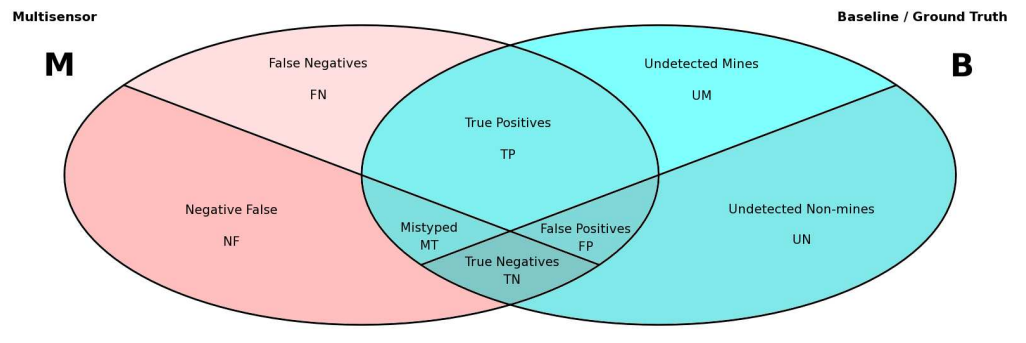

(b) Venn diagram

Figure 3. Basic principles and terminology needed when scoring results.

\subsection{Documentation and archiving}

Historically too little attention has been given to documentation and archiving of the results of multisensor test campaigns. To be able to track the performance of systems over longer time spans data and other ancillary results need to be stored or archived in proper fashion. Measurement data without proper documentation can be worthless if important information describing the sensor, the environment, position and location, and other factors are not available. In this respect the following recommendations are made:

- Test results and data must be maintained in accessible formats (also in the future and other platforms)

- A standardised table of content should be provided (i.e.template fo what should be included).

- A preference for concise and self-describing data format for storage of measurement data (e.g. netCDF, XML, etc...)

- Archival media have a limited 'shelf time'. Computer magazines and library sources suggest the following rules of thumb:

- short term (3-5 years): DVD and CDROM

- long term (5-15 years): hard drive (disconnected) 
A good candidate for storing scientific data and similar but simpler in concept to XML is netCDF [10] [11] [12]. This is a cross platform standard (both software and hardware) which is already widely used in areas like climatology, meteorology and modelling. Libraries enabling fast en efficient access to the data exist for many platforms. Interfaces for several high level programming languages exist. Many readily available viewers and browser are available. The format is well structured and self describing. It supports storage of really large data sets well beyond the traditional 2 GB limit with a maximum file size as large as $2^{63}$ bytes, or $8 \mathrm{~EB}$ (the underlying operating system also has to allow for this). The format is suitable for storing data of several different sensors. At TNO we have decided to use this format as a preferred format and have written code to support this. This will be made available though the ITEP website. Figure 4 show a schematic example of a netCDF used to store GPR data and other related data.

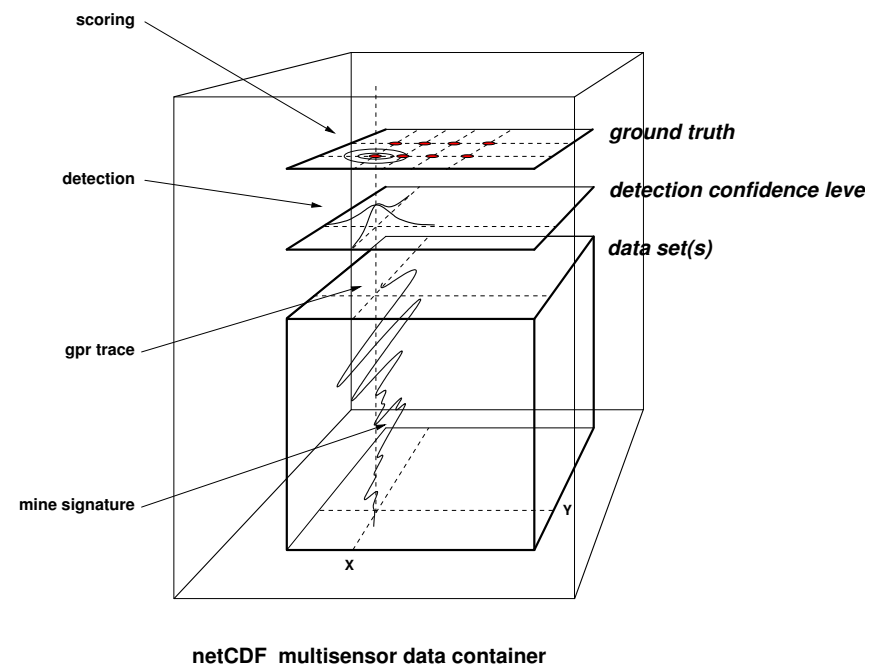

Figure 4. Schematic illustration of a sample use of netCDF

\section{CONCLUSIONS}

We have described briefly some of the issues that need to be addressed when drafting a so called "best practice" document. Undoubtedly there remain many omissions and oversights in our approach. At the moment the test that are executed concentrate on hand-held systems that are a combination of GPR and MD. We have tried to make our approach general enough to also cover vehicle based systems, that may also include other sensors like IR and acoustic. To compile a broadly accepted and widely applicable test document, input from many parties is needed, We therefore encourage manufacturers, researchers, deminers and other to actively contribute in this field.

The recommended approach emphasises :

Transparency The procedures should be clear and well enough standardised that essentially the same facility can be duplicated in other locations worldwide. Ideally it should be possible for system developers and manufacturers to predict fairly well the results of scoring their systems before actually undergoing tests.

Objectivity Human factors, other than inevitable training factors, should be isolated from the test environment so that results do not depend on a unique cadre of people with particular skills either in operating equipment or in processing the results.

Depth The range of tests should be sufficient to give insight not just into the detection performance of the system under test but also how that performance will depend on situational variables (weather, type of target, clutter environment, etc.)

Consensus We are attempting to define a set of testing protocols that will be useful to a wide range of government acquisition authorities, end-users, and manufacturers. To that end it is essential that each of these interest communities provide viewpoints to the definition process. 


\section{REFERENCES}

1. CEN, Humanitarian Mine Action - Test and Evaluation - Metal Detectors, CWA 14747 ed., 2003.

2. ITEP, "International test and evaluation program for humanitarian demining." web-site, http://www.itep.ws.

3. J. B. Rhebergen, "Mine-detection test facilities at TNO-FEL site 'Waalsdorp': Some examples of results obtained with an array based GPR system," Subsurface Sensing Technologies and Applications 3, pp. 369-385, October 2002.

4. M. Sato, Y. Hamada, X. Feng, F. Kong, Z. Zeng, and G. Fang, "GPR using and array antenna for landmine detection," Near Surface Geophysics 2, pp. 3-9, February 2004.

5. J. B. Rhebergen, H. A. Lensen, S. P. B. W., J. M. H. Hendrickx, and G. Rodríguez Marín, "Soil water distribution around landmines and the effect on dielectric contrast," in Detection and Remediation Technologies for Mines and Mine-like Targets VII, Proceedings of the SPIE, SPIE, April 2002.

6. H. J. M. H. Miller, T. W. and and B. Borchers, "Radar detection of buried landmines in field soils," Soil Science Society of America Journal, September 2002. submitted for publication.

7. W. de Jong, H. A. Lensen, and Y. H. L. Janssen, "Sophisticated test facility to detect landmines," in Detection and Remediation Technologies for Mines and Mine-like Targets IV, Proceedings of the SPIE 3710, pp. 1409-1418, SPIE, April 1999.

8. E. M. Rosen, "Testing and evaluation of forward looking GPR countermine systems," in Detection and Remediation Technologies for Mines and Mine-like Targets X, Proceedings of the SPIE 5794, SPIE, 2005.

9. J. Ishikawa, M. Kiyota, and F. Katsuhisa, "Experimental design for test and evaluation of anti-personnel landmine detection based on vehicle-mounted GPR systems," in Detection and Remediation Technologies for Mines and Minelike Targets X, Proceedings of the SPIE 5794, SPIE, 2005.

10. R. K. Rew and G. P. Davis, "NetCDF: An interface for scientific data access," in Computer Graphics and Applications, pp. 76-82, IEEE, July 1990.

11. S. A. Brown, M. Folk, G. Goucher, and R. Rew, "Software for portable scientific data management," in Computers in Physics, 7(3), American Institute of Physics, 1993.

12. UniData, "netCDF (network common data form." web-site, http://my . unidata.ucar. edu/content/software/ netcdf/index.html. 